\title{
USP8 mutations in corticotroph adenomas determine a distinct gene expression profile irrespective of functional tumour status
}

\author{
Mateusz Bujko1,*, Paulina Kober1,*, Joanna Boresowicz', Natalia Rusetska1, Agnieszka Paziewska2,6,

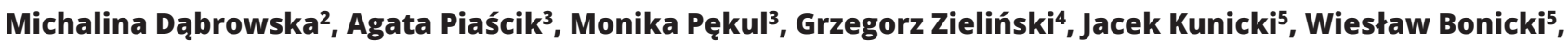 \\ Jerzy Ostrowski ${ }^{2,6}$, Janusz A Siedlecki ${ }^{1}$ and Maria Maksymowicz ${ }^{3}$
}

${ }^{1}$ Department of Molecular and Translational Oncology, ${ }^{2}$ Department of Genetics, ${ }^{3}$ Department of Pathology and Laboratory Diagnostics, Maria Sklodowska-Curie Institute - Oncology Center, Warsaw, Poland, ${ }^{4}$ Department of Neurosurgery, Military Institute of Medicine, Warsaw, Poland, ${ }^{5}$ Department of Neurosurgery, Maria Sklodowska-Curie Institute Oncology Center, Warsaw, Poland, and ${ }^{6}$ Department of Gastroenterology, Hepatology and Clinical Oncology, Medical Center for Postgraduate Education, Warsaw, Poland

Correspondence should be addressed to M Maksymowicz Email

mmaksymowicz@coi.waw.pl

\begin{abstract}
Objective: Pituitary corticotroph adenomas commonly cause Cushing's disease (CD) but part of these tumours are hormonally inactive (silent corticotroph adenomas, SCA). USP8 mutations are well-known driver mutations in corticotrophinomas. Differences in transcriptomic profiles between functioning and silent tumours or tumours with different USP8 status have not been investigated.

Design and methods: Forty-eight patients (28 CD, 20 SCA) were screened for USP8 mutations with Sanger sequencing. Twenty-four patients were included in transcriptomic profiling with Ampliseq Transcriptome Human Gene Expression Core Panel. The entire patients group was included in qRT-PCR analysis of selected genes expression. Immunohistochemistry was used for visualization of selected protein.

Results: We found USP8 mutation in 15 patients with CD and 4 SCAs. USP8 mutations determine molecular profile of the tumours as showed by hierarchical clustering and identification of 1648 genes differentially expressed in USP8mutated and USP8-wild-type tumours. Mutations affect many molecular pathways as observed in Gene Set Enrichment analysis. USP8-mutated adenomas showed higher level of POMC, CDC25A, MAPK4 but lower level of CCND2, CDK6, CDKN1B than USP8-wt tumours. Eighty-seven genes differentially expressed between CD-related adenomas and SCAs were found, including those involved in cell signalling (GLI2, DLC1, TBX2, RASSF6), cell adhesion (GJA1, CDH6), ion transport (KCNN4, KCNJ5) and GABA signalling (GABBR2, GABRD).

Conclusion: USP8 mutations occur in functioning and silent corticotrophinomas. They have pleiotropic effect, not limited to EGFR signalling, and affect expression levels of many genes involved in different pathways. Expression of GABA-related genes GABBR2, GNAL, GABARD and KCNJ5 correspond to functional status of the tumours.
\end{abstract}

\section{Introduction}

Pituitary tumours represent about $10-20 \%$ of all intracranial neoplasms and over 90\% of tumours are located in the sella turcica; their prevalence in the population is estimated to be approximately $1 / 1000$. These (c) 2019 European Society of Endocrinology Printed in Great Britain tumours may arise from different kinds of secretory cells of pituitary gland, and therefore, they are characterized by overproduction of certain hormones which determine the clinical manifestation of the disease.

Published by Bioscientifica Ltd. 
Corticotroph adenomas commonly cause ACTHdependent Cushing's disease (CD) a severe, life-threatening disorder. However, a proportion of tumours originating from corticotrophic pituitary cells are hormonally nonfunctioning (1). They do not affect hormonal balance, do not cause CD symptoms and are classified as silent corticotroph adenomas (SCAs). Similar to most patients with non-functioning pituitary adenomas (NFPAs), SCA patients are diagnosed due to neurological symptoms (for example visual impairment) when tumours reach macroadenoma size $(>10 \mathrm{~mm})$. Corticotrophic nature of the tumours is confirmed in histopathological examination based on immunohistochemical staining. SCAs are commonly diagnosed at macroadenoma stage and represent one of the most aggressive subtypes of pituitary tumours characterized by invasive growth and high relapse rate (1).

Recently, notable progress in the understanding of molecular background of CD has been made (2) including the discovery of recurrent USP8 hot spot mutation $(3,4,5)$. In contrary, the biology of SCA still remains unexplored. The reason why some ACTH-producing adenomas (as confirmed by immunohistochemistry and electron microscopy) remain endocrinologically inactive is still unclear and the results of very few studies in this field were published $(6,7,8,9,10,11,12,13)$. Similarly, the molecular basis of aggressive growth of these tumours is very poorly understood $(14,15,16,17)$ and research on SCAs and CD-causing tumours using genome-wide molecular techniques is lacking.

The aim of the study was to compare gene expression profiles of functioning and SCAs in order to identify a biological mechanism contributing to distinct hormonal secretory activities. Since USP8 is a well-recognized driver gene mutated in large proportion of corticotrophinomas, we intended to verify whether its mutations occur also in SCAs. Moreover, we intended to verify how USP8 mutations affect transcriptomic profiles of $\mathrm{CD}$ and SCA.

\section{Subjects and methods}

\section{Patients and samples}

Pituitary tumour samples from 48 patients were collected during transsphenoidal surgery and fixed in formalin for routine diagnostic procedures. Diagnoses were based on WHO 2004 criteria. Archival FFPE tissue samples, including 28 samples from patients with $\mathrm{CD}$ and 20 samples of SCA from years 2013 to 2017 were chosen and reevaluated histopathologically.
The diagnosis of Cushing's syndrome/ hypercortisolemia was based on standard hormonal criteria: increased urinary free cortisol (UFC) in three 24-h urine collections, disturbances of cortisol circadian rhythm, increased serum cortisol levels accompanied by increased or not suppressed plasma ACTH levels at 8:00 h, and a lack of suppression of serum cortisol levels to $<1.8 \mu \mathrm{g} / \mathrm{dL}$ during an overnight dexamethasone suppression test (1 $\mathrm{mg}$ at midnight). The pituitary aetiology of $\mathrm{CD}$ was confirmed based on the serum cortisol levels or UFC suppression $<50 \%$ with a high-dose dexamethasone suppression test ( $2 \mathrm{mg}$ q.i.d. for $48 \mathrm{~h}$ ) or a positive result of a corticotrophin-releasing hormone stimulation test (100 mg i.v.) and positive pituitary magnetic resonance imaging. In the group of SCAs, none of the patients had any evidence of hypercortisolemia based on clinical signs and symptoms as well as basic laboratory tests. ACTH levels were assessed using IRMA (ELSA-ACTH, CIS Bio International, Gif-sur-Yvette Cedex, France). The analytical sensitivity was $2 \mathrm{pg} / \mathrm{mL}$ (reference range: $10-60 \mathrm{pg} / \mathrm{mL}$ ). Serum cortisol concentrations were determined by the Elecsys 2010 electrochemiluminescence immunoassay (Roche Diagnostics). Analytical sensitivity of the assay was $0.02 \mu \mathrm{g} / \mathrm{dL}$ (reference range: $6.2-19.4 \mu \mathrm{g} / \mathrm{dL}$ ). UFC was determined after extraction (liquid/liquid with dichloromethane) by electrochemiluminescence immunoassay (Elecsys 2010, Roche Diagnostics) reference range: $4.3-176 \mu \mathrm{g} / 24 \mathrm{~h}$.

Characteristics of the patients are presented in Table 1. The study was approved by the Local Ethics Committee of Maria Sklodowska-Curie Institute - Oncology Center in Warsaw, Poland. Each patient provided informed consent for the use of tissue samples for scientific purposes. The content of tumour tissue in each FFPE sample ranged between 80 and 100\% (median 99\%), as described in detail in Supplementary Table 1 (see section on supplementary data given at the end of this article).

DNA and total RNA from FFPE samples was isolated using RecoverAll ${ }^{\mathrm{TM}}$ Total Nucleic Acid Isolation Kit for FFPE (Thermo Fisher Scientific) and measured using NanoDrop 2000 (Thermo Fisher Scientific). The samples were stored at $-70^{\circ} \mathrm{C}$.

\section{Assessment of USP8 mutation status}

The presence of point mutation at USP8 hot spot (exon 14) was determined using Sanger sequencing. DNA was PCR amplified with FastStart Taq DNA Polymerase (Roche Diagnostics) using GeneAmp 9700 PCR system (Applied Biosystems). The PCR product was purified using ExoStar 
Table 1 Patients' characteristics.

\begin{tabular}{|c|c|c|}
\hline & CD & SCA \\
\hline Number of patients & 28 & 20 \\
\hline Age (years; median (range)) & $43(23-76)$ & $53(23-77)$ \\
\hline \multicolumn{3}{|l|}{ Gender } \\
\hline Male & 3 & 9 \\
\hline Female & 25 & 11 \\
\hline \multicolumn{3}{|l|}{ Ultrastructural characteristics } \\
\hline Sparsely granulated & 9 & 12 \\
\hline Densely granulated & 19 & 8 \\
\hline \multicolumn{3}{|l|}{ KNOSP grade } \\
\hline 0 & 5 & 2 \\
\hline 1 & 12 & 9 \\
\hline 2 & 4 & 5 \\
\hline 3 & 3 & 2 \\
\hline 4 & 4 & 2 \\
\hline \multicolumn{3}{|l|}{ Tumour size } \\
\hline Macroadenoma & 17 & 20 \\
\hline Microadenoma & 11 & 0 \\
\hline USP8 mutation & 11 & 4 \\
\hline
\end{tabular}

(GE Healthcare Life Sciences), labelled with BigDye Terminator v.3.1 (Applied Biosytems) according to the manufacturer's instructions and analysed by capillary electrophoresis with the ABI PRISM 3300 Genetic Analyzer (Applied Biosystems). PCR primers sequences are presented in Supplementary Table 2. NM_005154.5 (NP_005145.3) sequence was used as reference.

\section{High-throughput transcriptome profiling with next-generation sequencing}

RNA isolated from FFPE samples was used for ampliconbased library preparation and ion semiconductor sequencing. Human transcriptome sequencing library was prepared using the Ion AmpliSeq ${ }^{\text {TM }}$ Transcriptome Human Gene Expression Kit (Thermo Fisher Scientific) according to the manufacturer's protocol. Briefly, 100 ng of total RNA was subjected to multiplex amplification with Ion AmpliSeq Transcriptome Human Gene Expression Core Panel (Thermo Fisher Scientific). Primer sequences of amplicons were digested and adapters were ligated to the amplicons. The samples were then purified using Agencourt AMPure XP beads (Beckman Coulter) and stored at $-20^{\circ} \mathrm{C}$ for further processing. The concentration and DNA fragment length of each library was determined using Agilent High Sensitivity DNA Analysis Kits on a Bioanalyzer 2100 (Agilent). Each library was diluted to $\sim 55 \mathrm{pM}$ prior to template preparation. Up to seven barcoded libraries were subjected to automated template preparation with the Ion PI Hi-Q Chef Kit on the Ion Chef Instrument, which performs emulsion PCR on Ion Sphere Particles, followed by particle recovery and template loading on a PI chip. Samples were sequenced in the Ion Proton instrument on a PI chip using the sequencing reagents (Ion PI ${ }^{\mathrm{TM}} \mathrm{Hi}-\mathrm{Q}^{\mathrm{TM}}$ Sequencing $200 \mathrm{Kit}$ ) provided as part of the Ion PI ${ }^{\mathrm{TM}} \mathrm{Hi}-\mathrm{Q}^{\mathrm{TM}}$ Chef Kit according to the manufacturer's instructions (Thermo Fisher Scientific).

All the steps of data analysis were performed using Bioconductor packages in $\mathrm{R}$ programming language environment. The raw un-normalized count matrix was generated from BAM files using Genomic Alignments package (18). Then it was imported to DESeq2 which performed the necessary normalization and calculated estimates of dispersion (19), and then low-expression gene filtration was applied (the genes with at least five sequencing reads in at least half of the samples were included) and subsequent analysis of differentially expressed genes between groups by use of discrete distributions and negative binomial generalized linear models was performed. Fold change of expression (FC), calculated as read counts ratio USP8-mutated (USP8mut)/USP8-wild type (USP8wt) or CD/SCA was used as a measure of expression difference between the groups of samples. Differentially expressed genes (DEGs) were defined as those with adjusted $P$ value $<0.05$. DEGs with FC $>2.5$ or FC $<0.4$ were used for Gene Set Enrichment analysis (GSEa).

\section{Gene expression assessment using qRT-PCR}

Expression levels of the selected genes were assessed using qRT-PCR. One microgram of each FFPE tissue-derived RNA sample was subjected to reverse transcription using the Transcriptor First Strand cDNA Synthesis Kit (Roche Diagnostics). qRT-PCR was carried out using a 384-well format and the 7900HT Fast Real-Time PCR System (Applied Biosystems) with Power SYBR Green PCR Master Mix (Thermo Fisher Scientific) in a volume of $5 \mu \mathrm{L}$, containing $2.25 \mathrm{pmol}$ of each primer. The samples were amplified in triplicates. GAPDH was used as reference gene. Standard curves, based on amplification of known cDNA template concentrations, were used for determining PCR efficiency. Delta Ct method was used to calculate relative expression level. PCR primers' sequences are presented in Supplementary Table 2. PCR nondetects were used in calculation of relative gene expression level with the use of nondetects R package (20).

\section{Immunohistochemical staining}

Immunohistochemical staining (IHC) was performed on 4- $\mu \mathrm{m}$ FFPE tissue sections from 24 samples (12 SCA and 
12 CD tumours) using the Envision Detection System (Dako). Sections were deparaffinized with xylene and rehydrated in a series of ethanol solutions of decreasing concentration. Heat-induced epitope retrieval was carried out in a Target Retrieval Solution $\mathrm{pH} 9$ (Dako) in a $96^{\circ} \mathrm{C}$ water bath, for $20 \mathrm{~min}$. After cooling the retrieval solutions for $25 \mathrm{~min}$ at room temperature, the slides were treated for 5 min with a Blocker of Endogenous Peroxidase (Dako). Slides were incubated overnight at $4^{\circ} \mathrm{C}$ with polyclonal antibody anti-KCNJ5 (PA5-53594, Thermo Fisher Scientific) (dilution 1:50) and subsequently labelled with the Envision Detection System (Dako). The colour reaction product was developed with 3,3'-diaminobenzidine tetrahydrochloride (Dako) as a substrate, and nuclear contrast was achieved with hematoxylin counterstaining. Analysis of immunohistochemical reactivity was performed by calculating H-score, which combines information on both reaction intensity (scored from 0 to 3 ) and number of the cells with a given intensity. Previously reported formula was used for quantification (21). Scoring results were analysed as continuous variables.

\section{Statistical analysis}

Quantitative continuous variables were analysed using the two-sided Mann-Whitney $U$ test. Two-sided Fisher's exact test was used for the analysis of proportions. A significance threshold level $\alpha=0.05$ was used. Data were analysed and visualized using GraphPad Prism (GraphPad Software). Hierarchical clustering using the values for all the genes that passed the filtration was done using hclust function, (stats $\mathrm{R}$ package). Complete-linkage clustering with Euclidean distance as similarity metrics was used. EnrichR tool was used for Gene Set Enrichment (GSE) analysis (22). The GSE terms meeting the criterion of adjusted $P<0.05$ were considered as significantly enriched. Cluster 3.0 and TreeView 1.6 software (Stanford University School of Medicine, Stanford, CA, USA) were used for heat map preparation.

\section{Results}

\section{Prevalence of USP8 mutation in patients}

Hot spot mutations in exon 14 of USP8 were found in 15 patients including 11/28 (39.2\%) of CD adenomas and 4/20 (20\%) SCAs. The mutations were missense variants: p.Pro720Arg (five patients), p.Pro720Gln (one patient), p.Ser718Pro (two patients) and in-frame deletion p.Ser718del (seven patients). All the mutations were heterozygous. The results of Sanger sequencing are presented in Supplementary Fig. 1. The observed prevalence of mutations was higher in CD than in SCA patients, but the difference did not reach significance threshold level $(P=0.2122)$. No difference in the frequency of USP8 mutations was observed in patients stratified according to results of ultrastructural examination of the tumours (USP8 mutation was detected in 10/27 of densely granulated and in 5/21 of sparsely granulated adenomas, $P=0.366$ ) and sex of the patients (mutation was detected in $1 / 12$ man and $14 / 36$ women; $P=0.0729$ ). No difference in age of the USP8mut and USP8wt patients was found (mean age 43.8 vs 50.8 , respectively; $P=0.1220$ ).

\section{Effect of USP8 mutation on gene expression profile}

Twenty-four patients including 12 CD patients (6 USP8mut and 6 USP8wt) as well as 12 SCA patients (4 USP8mut and 8 USP8wt) were subjected to gene expression profiling with the use of Ion AmpliSeq Transcriptome Human Gene Expression Core Panel. Following data filtration and normalization, 17550 human genes were subjected to analysis that involved hierarchical clustering and identification of genes differentially expressed between functioning and silent corticotrophinomas as well as between USP8mut and USP8wt tumours. PCA analysis performed on read counts normalized for library size showed four outlier samples (Supplementary Fig. 2 ). These samples were excluded from the analysis to avoid technical bias. The data were deposited in NCBI's Gene Expression Omnibus and are accessible through GSE132982 accession number.

Hierarchical clustering of samples based on whole transcriptional data revealed that USP8mut tumours represent a separate gene expression cluster, irrespective of their endocrinological status (Fig. 1A). The predominant role of USP8 mutations in defining the phenotype of corticotroph adenomas was confirmed by identification of 1648 DEGs differentiating USP8mut and USP8wt tumours (adjusted $P$ value $<0.05$ ). These DEGs, including 595 genes with $\mathrm{FC}>2.5$ or $\mathrm{FC}<0.4$, are presented in Fig. $1 \mathrm{~B}$ and listed in Supplementary Table 3. Using Gene Ontology (GO) analysis as well as Panther and Reactome Pathways databases with EnrichR tool (22), we sought the molecular pathways represented by these 595 DEGs by GSE analysis. We identified 11 GO terms enriched with DEGs related to few general processes: nervous system development and functioning, nucleosome organization, cell-cell adhesion and regulation of cell 
A

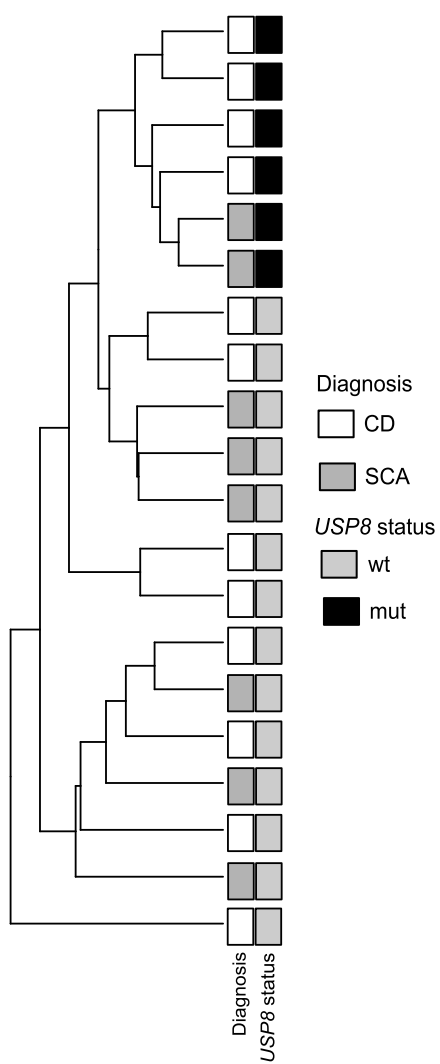

B

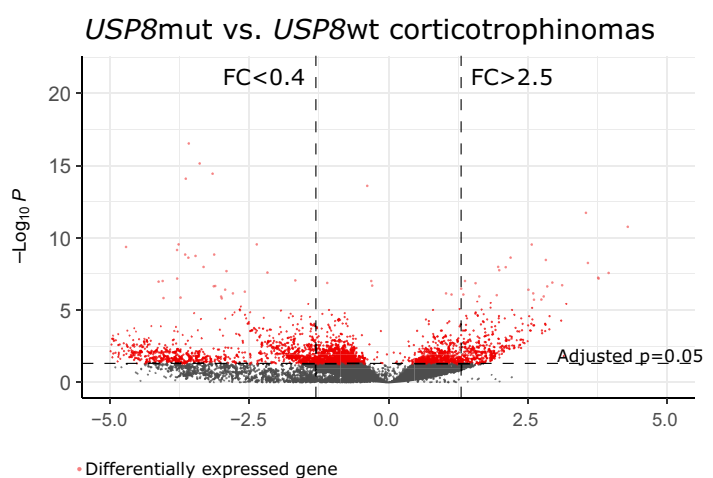

Silent vs. functioning corticotrophinomas

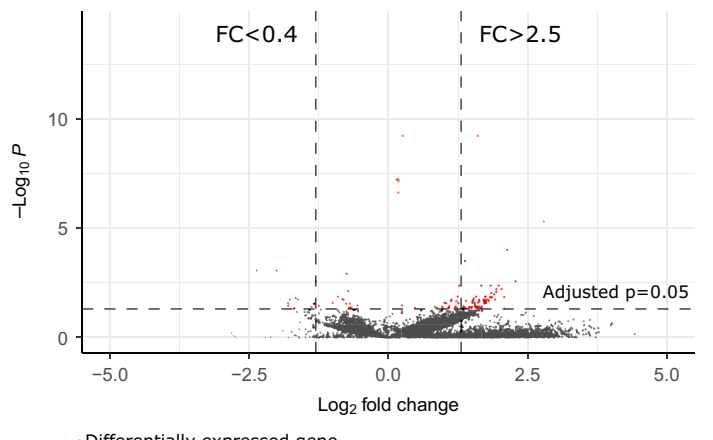

C

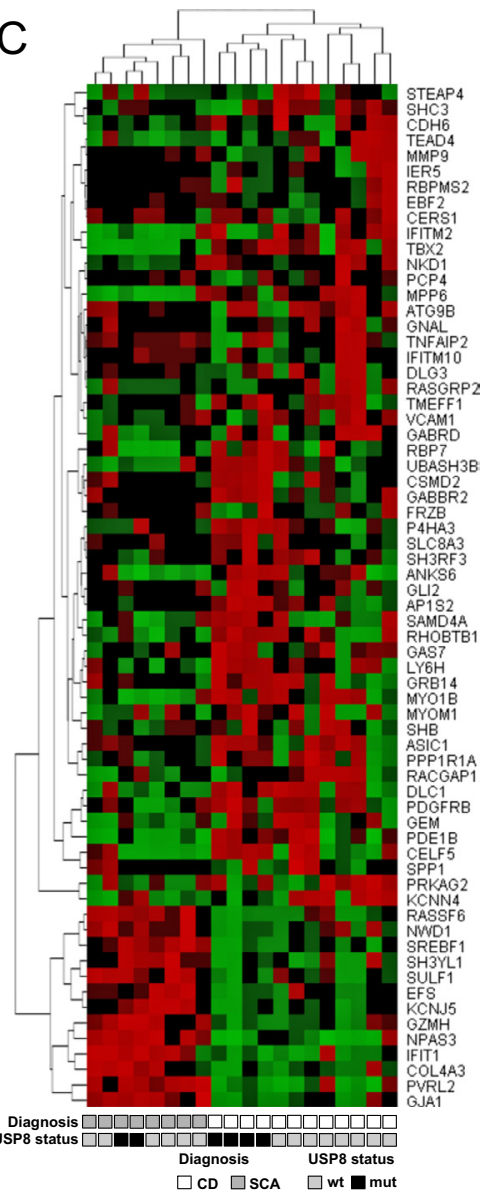

\section{Figure 1}

Results of transcriptomic profiling of corticotroph tumours with regards to USP8 mutation and functional status. (A) Hierarchical clustering of tumour samples based on the transcriptional profile (17 550 genes). (B) Volcano plots presenting distribution of DEGs identified in comparisons of USP8mut vs USP8wt tumours as well as SCAs vs functioning corticotrophinomas. (C) Expression of genes differentially expressed in SCAs and adenomas causing Cushing's disease with FC $>2.5$ or FC $<0.4$.

proliferation. Two Panther pathways were identified as significantly enriched: cadherin signalling and Wnt signalling pathways. Seventy-three reactome pathways were identified as significantly enriched. Most of the pathways were identified due to the high representation of genes encoding replication-dependent histone variants that are expressed at significantly lower level in USP8mut tumours. Top GSE results are presented in Table 2, while details are shown in Supplementary Table 3.

\section{Gene expression in functioning and silent corticotrophinomas}

When comparing silent and functional corticotrophinomas, eighty-seven differentially expressed genes were found (Supplementary Table 4) including 66 genes with $\mathrm{FC}>2.5$ or $\mathrm{FC}<0.4$ that are presented in Fig. $2 \mathrm{~B}$. These genes encode functionally diverse proteins that play a role in different cellular processes including cell signalling (GLI2, DLC1, TBX2, RASSF6, GNAL, RASGRP2, PDGFRB, GABBR2), cell adhesion (GJA1, CDH6), ion channels functioning (KCNN4, KCNJ5, GABRD) and others. The highest fold change of expression was observed for KCNJ5 (most upregulated in SCAs) and GABBR2 (most downregulated in SCAs) genes that both belong to a GABAB receptor pathway according to Reactome db. GSE analysis with Reactome database showed GABAB receptor activation (Reactome pathways R-HSA-991365 and R-HSA977444) as the only pathway significantly enriched for the differentially expressed genes (Table 2 and Supplementary Table 4). Three DEGs were annotated to these pathways: GABBR2, KCNJ5 and GNAL. No significant enrichment was 
Table 2 The results of Gene Set Enrichment analysis for genes differentially expressed in USP8-mutated and USP8 wild-type tumours.

\begin{tabular}{|c|c|c|c|}
\hline & Term & Overlap & Adjusted $P$ \\
\hline \multirow{2}{*}{\multicolumn{4}{|c|}{$\begin{array}{l}\text { GSE analysis for genes differentially expressed in USP8-mutated and USP8 wt patients } \\
\text { GO molecular processes }\end{array}$}} \\
\hline & & & \\
\hline 1 & Synapse assembly (GO:0007416) & $16 / 64$ & $<0.0001$ \\
\hline 2 & Nucleosome assembly (GO:0006334) & $10 / 61$ & 0.0092 \\
\hline 3 & Anterograde trans-synaptic signalling (GO:0098916) & $21 / 241$ & 0.0092 \\
\hline 4 & Chromatin assembly (GO:0031497) & $10 / 66$ & 0.0096 \\
\hline 5 & Nervous system development (GO:0007399) & $31 / 456$ & 0.0096 \\
\hline 6 & $\begin{array}{l}\text { Cell-cell adhesion via plasma-membrane adhesion } \\
\text { molecules (GO:0098742) }\end{array}$ & $15 / 143$ & 0.0096 \\
\hline 7 & Chemical synaptic transmission (GO:0007268) & $22 / 290$ & 0.0208 \\
\hline 8 & Nucleosome organization (GO:0034728) & $10 / 80$ & 0.0381 \\
\hline 9 & $\begin{array}{l}\text { Negative regulation of epithelial cell proliferation } \\
\text { (GO:0050680) }\end{array}$ & $9 / 67$ & 0.0419 \\
\hline 10 & Extracellular matrix organization (GO:0030198) & $18 / 230$ & 0.0453 \\
\hline \multicolumn{4}{|c|}{ Panther pathways } \\
\hline 1 & Cadherin signalling pathway_Homo sapiens_P00012 & $17 / 150$ & 0.0002 \\
\hline 2 & Wnt signalling pathway_Homo sapiens_P00057 & $20 / 278$ & 0.0088 \\
\hline \multicolumn{4}{|c|}{ Reactome pathways } \\
\hline 1 & $\begin{array}{l}\text { RNA Polymerase I Promoter Opening_Homo } \\
\text { sapiens_R-HSA-73728 }\end{array}$ & $10 / 30$ & $<0.0001$ \\
\hline 2 & Packaging Of Telomere Ends_Homo sapiens_R-HSA-171306 & $10 / 31$ & $<0.0001$ \\
\hline 3 & DNA methylation_Homo sapiens_R-HSA-5334118 & $10 / 32$ & $<0.0001$ \\
\hline 4 & $\begin{array}{l}\text { Condensation of Prophase Chromosomes_Homo sapiens_R- } \\
\text { HSA-2299718 }\end{array}$ & $11 / 41$ & $<0.0001$ \\
\hline 5 & $\begin{array}{l}\text { DNA Damage/Telomere Stress Induced Senescence_Homo } \\
\text { sapiens_R-HSA-2559586 }\end{array}$ & $13 / 59$ & $<0.0001$ \\
\hline 6 & $\begin{array}{l}\text { Activated PKN1 stimulates transcription of AR (androgen } \\
\text { receptor) regulated genes KLK2 and KLK3_Homo } \\
\text { sapiens_R-HSA-5625886 }\end{array}$ & $10 / 34$ & $<0.0001$ \\
\hline 7 & $\begin{array}{l}\text { SIRT1 negatively regulates rRNA Expression_Homo } \\
\text { sapiens_R-HSA-427359 }\end{array}$ & $10 / 35$ & $<0.0001$ \\
\hline 8 & Meiotic recombination_Homo sapiens_R-HSA-912446 & $12 / 54$ & $<0.0001$ \\
\hline 9 & $\begin{array}{l}\text { PRC2 methylates histones and DNA_Homo } \\
\text { sapiens_R-HSA-212300 }\end{array}$ & $10 / 40$ & $<0.0001$ \\
\hline 10 & Cellular Senescence_Homo sapiens_R-HSA-2559583 & $19 / 161$ & $<0.0001$ \\
\hline \multicolumn{4}{|c|}{$\begin{array}{l}\text { GSE analysis for genes differentially expressed in SCAs and functioning corticotrophinomas } \\
\text { Reactome pathways }\end{array}$} \\
\hline 1 & Activation of GABAB receptors_Homo sapiens_R-HSA-991365 & $3 / 39$ & 0.0003 \\
\hline 2 & GABA B receptor activation_Hōmo sapiens_R-HSA-977444 & 3/39 & 0.0003 \\
\hline
\end{tabular}

Up to top ten pathways are presented.

found in GSE analysis based on GO biological processes and Panther pathways.

\section{Evaluation of the expression level of selected genes with qRT-PCR}

The expression levels of 12 genes were assessed in 48 adenoma tumour samples with qRT-PCR. This set included POMC gene not included in Ion AmpliSeq Transcriptome Human Gene Expression Core Panel, USP8, EGFR, selected genes identified as differentially expressed in USP8mut and USP8wt tumours (CCND2, CDK6, CDKN1B, $C D C 25 A$ and MAPK4) as well as the genes related to GABA signalling (KCNJ5, GABBR2, GNAL and GABARD) (found as differentially expressed in SCA and CD patients).

USP8mut tumours revealed slightly higher POMC expression than USP8wt tumours (Fig. 2A), while no difference in the expression level of EGFR and USP8 was found between these tumours (Supplementary Fig. 3). Higher levels of $C C N D 2, C D K 6$ and $C D K N 1 B$ were observed in USP8wt tumours, CDC25A and MAPK4 expression level were higher in samples with USP8 mutation.

$P O M C$ expression was found lowered in nonfunctioning adenomas as compared to $\mathrm{CD}$; however, decreased expression was observed predominantly in USP8wt tumours. 
A

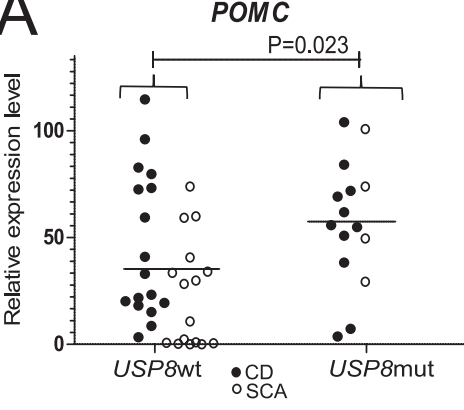

CDKN1B

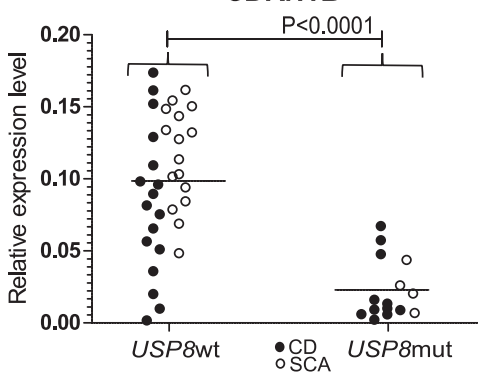

B
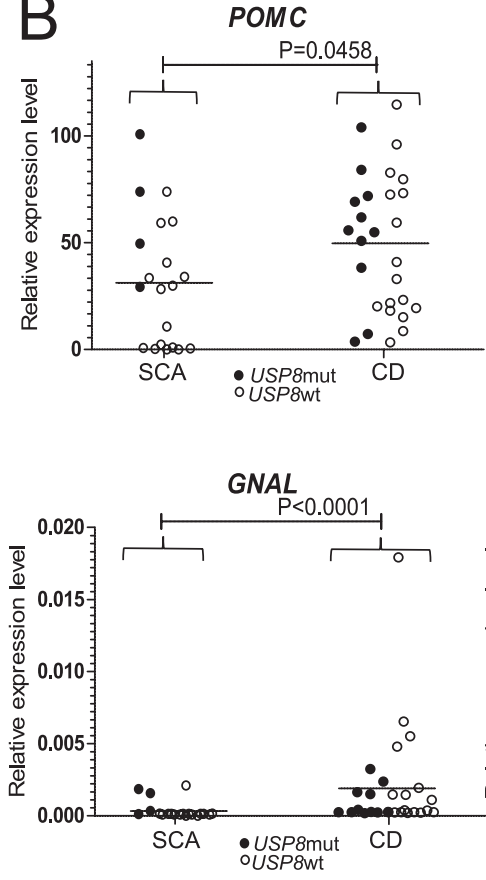

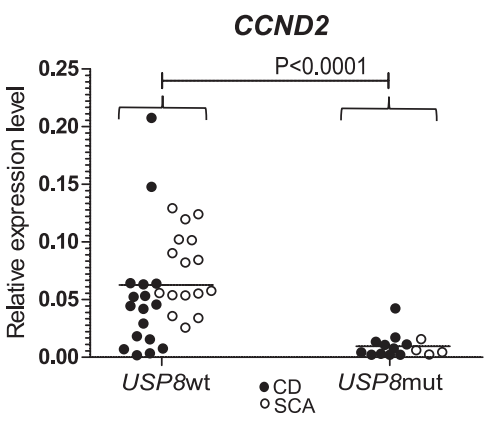

CDC25A

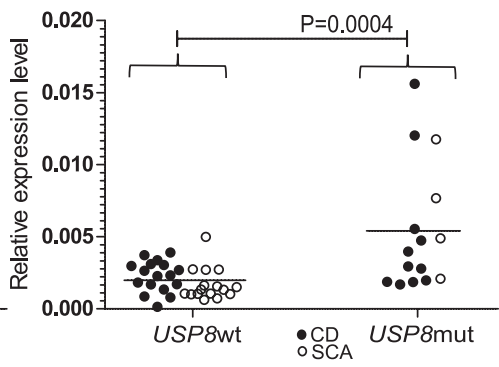

KCNJ5
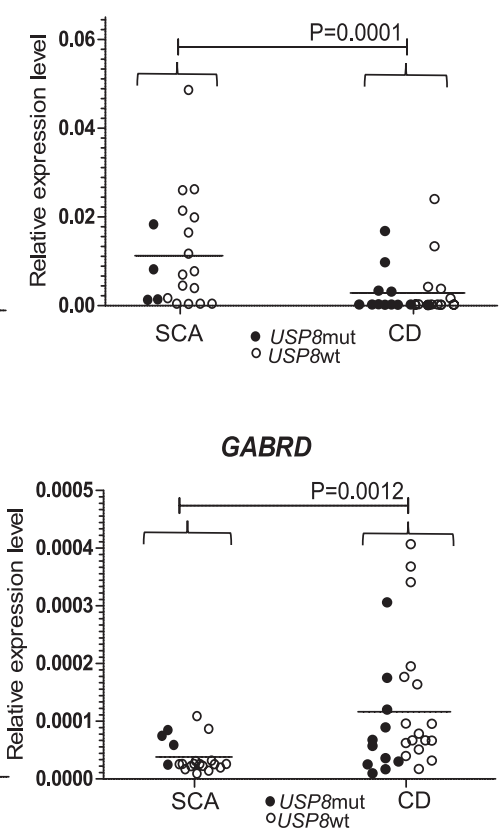

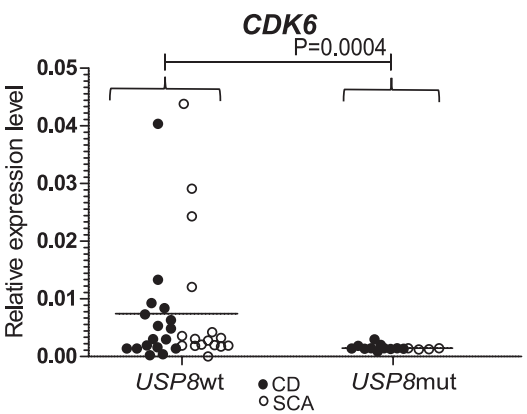

MAPK4

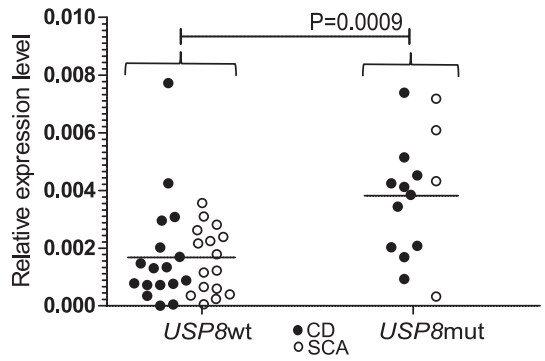

GABBR2

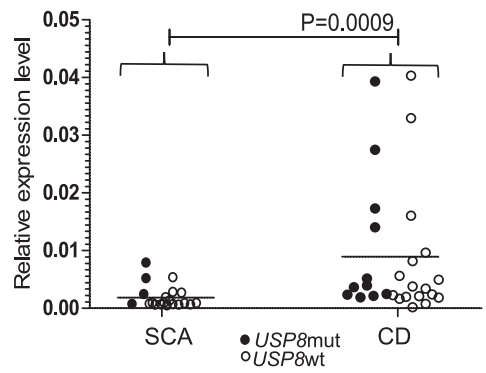

C

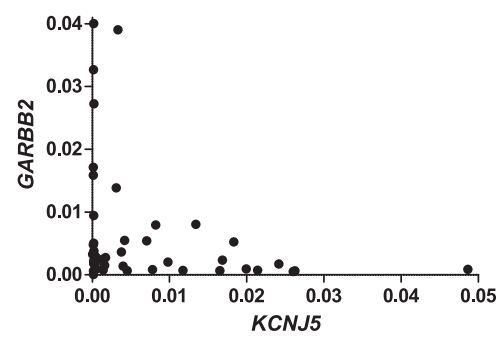

\section{Figure 2}

The results of gene expression measurements with qRT-PCR. (A) Comparison of gene expression levels in corticotroph tumours with USP8 mutation and wild-type corticotroph tumours. (B) Comparison of gene expression levels in SCA and functioning corticotroph tumours with regard to USP8 mutation status. (C) Spearman correlation between GABBR2 and KCNJ5 expression level.

The expression of KCNJ5 was higher in SCA patients than in patients with Cushing's disease, whereas mRNA levels of GABBR2, GNAL and GABRD were higher in CD patients (Fig. 2B), in line with the results of NGS-based transcriptomic profiling. Since KCNJ5, GABBR2 and $G N A L$ encode proteins involved in the same pathway, we examined the correlation between these genes expression levels. This analysis showed a negative correlation between 
KCNJ5 and GABBR2 (Spearman $R=-0.37 ; P=0.0095$ ) as shown in Fig. 2C.

\section{Immunohistochemical evaluation of KCNJ5 protein expression}

IHC was performed on 22 FFPE samples (11 SCA and $11 \mathrm{CD}$ tumours). Both membrane and cytoplasmic immunoreactivity was observed in 7/11 SCA samples and 4/11 adenomas causing CD (differences in the proportions did not reach significance threshold level). Nine tissue samples revealed only cytoplasmic immunoreactivity. The examples of tissue staining are presented in Fig. 3. H-score was calculated for all 22 patients and comparison of silent and functioning corticotrophinomas was performed. SCA samples showed higher immunostaining reactivity score as compared to functioning tumours with mean H-core 146 and 82.2 in SCA and CD patients, respectively $(P=0.0104$; Fig. 3$)$. Positive correlation between H-score and expression level of KCNJ5 measured with qRT-PCR was observed (Spearman $R=0.447 ; P=0.0368$ ).

\section{Discussion}

The frequency of USP8 mutations in CD patients observed in our study is similar to previously reported results where the mutations were identified in about $30-40 \%$ of the tumours $(3,5,23,24)$. However, both lower and higher mutations frequencies were also reported in other cohorts $(4,25,26)$. In our study, all the identified USP8 mutations were single amino acid substitutions or single codon deletion in hot spot region in exon 14 . All the alterations were also identified previously $(4,5,23,24,27)$.

Interestingly, in contrary to previous studies we also found USP8 mutations in SCA patients $(4 / 20)$. In the published reports $(5,26)$ no mutations were observed in non-functioning corticotroph tumours, however, a smaller number of SCA samples (11 and 2 samples) were included. After careful verification of clinical data to exclude misdiagnosis of these patients, we confirmed all the cases to meet criteria for SCAs. Regardless of diagnostic criteria, the biological differences between SCAs and functioning corticotroph tumours are still unclear (1).

\section{A Functioning Corticotroph Tumors}
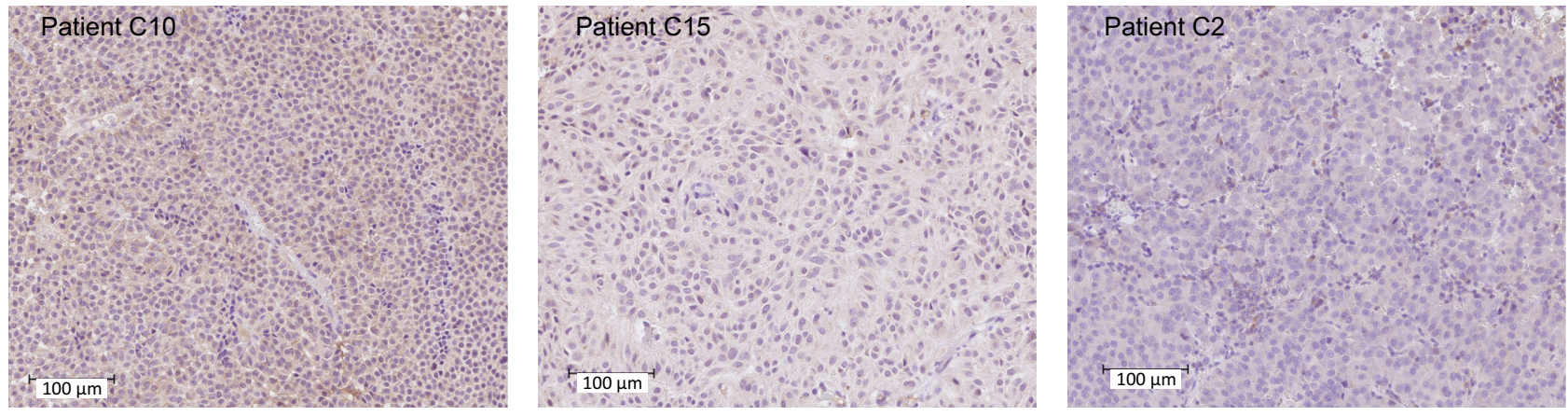

\section{B Silent Corticotroph Tumors}
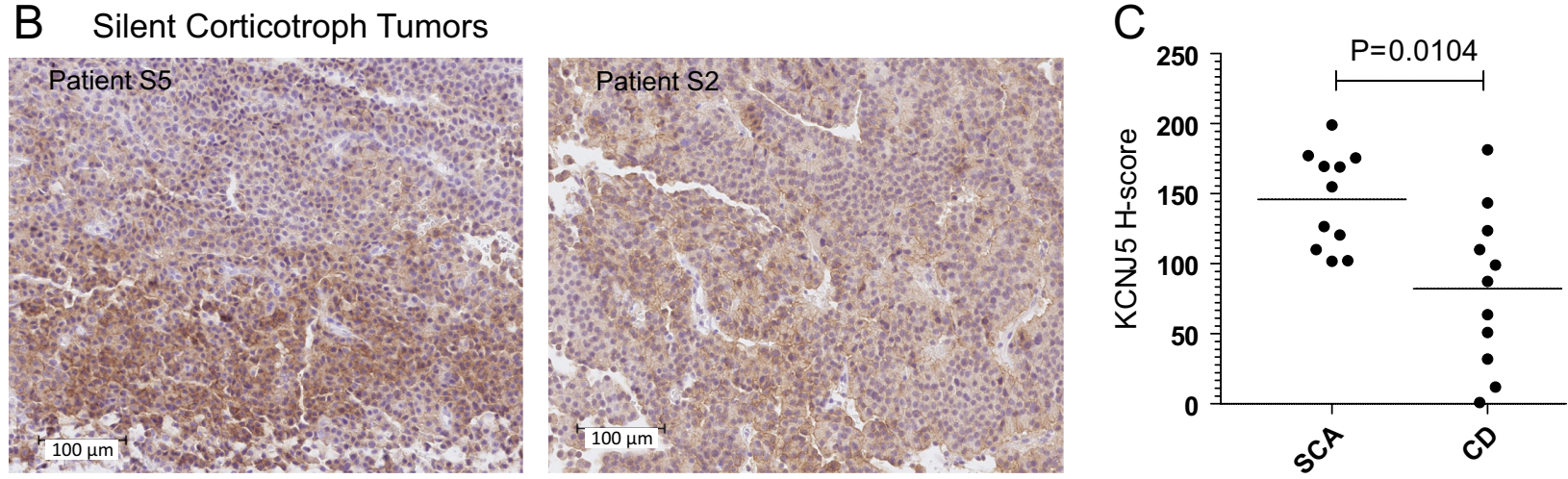

\section{Figure 3}

The results of immunohistochemical KCNJ5 detection in tissue samples. (A) The examples of immunostaining in functioning and silent corticotroph tumours. (B) The results of immunohistochemical staining evaluation. Comparison of $\mathrm{H}$-score values in silent and functioning corticotroph tumours. A full colour version of this figure is available at https://doi.org/10.1530/EJE-19-0194. 
Both tumour types develop from the same pituitary cell lineage and the evidence of SCA to CD conversion and vice versa was also reported $(8,28)$. Our results may suggest that USP8mut SCAs may have different molecular profile of gene expression than USP8wt SCAs, including higher POMC expression. However, this issue was not investigated due to a low number of patients.

The gene expression profiling of corticotroph adenomas clearly showed that USP8 mutations strongly affect molecular profile of tumours regardless of functional status. Hierarchical clustering based on whole transcriptomic data showed that USP8mut samples represent a separate cluster. In terms of genes expression, these samples are more similar to each other than samples from functional groups (silent and functioning corticotrophinomas). Presence of the mutations affects the expression of numerous genes involved in many different molecular processes and pathways. Processes related to cell-cell adhesion, cadherin signalling and Wnt signalling were found to be affected by USP 8 mutation status. These pathways were identified as significantly enriched due to the high representation of protocadherin-encoding genes and FZD1 in DEGs.

A large number of different pathways in GSE analysis with reactome database were found due to high representation of genes encoding replication-dependent histone variants among DEGs (with lower expression in USP8mut patients). More than half of all histone-encoding genes (29/49) that passed the filtration in the analysis of NGS results are differentially expressed. Alteration in the expression of this class of genes causes imbalance in the composition of histones by affecting chromatin organization and functional status (29). This may lead to disturbances in various cellular processes. Deregulation of histone-encoding genes' expression was described in carcinogenesis (29).

Cell cycle regulators have been recognized as involved in development of corticotroph tumours (30). In line with this are our findings of identification of DEGs among known cancer-related genes involved in the regulation of cell proliferation. Negative regulation of epithelial cell proliferation was identified among the enriched GO processes in GSE analysis due to differential expression of genes including CDK6, TGFB2, SOX2, SOX9, and SNAI2. As also further confirmed by qPCR evaluation, CCND2 (encoding cyclin D2) and CDK6 expression was higher in USP8wt tumours, suggesting increased cyclin D signalling in these tumours. In USP8mut tumours we observed low level of CDKN1B (encoding p27/Kpi1 inhibitor) and high expression of $C D C 25 A$, a known cell cycle-related oncogene (31). Correspondingly, a difference in p27/Kip1 protein level in mutated and wild-type corticotrophinoma patients was reported recently (27). MAPK4 (encoding ERK4) with higher expression in USP8mut tumours is atypical MAP kinase that was found to be overexpressed in human cancers. Its overexpression results in the increased cell proliferation through stimulation of AKT/ mTOR signalling (32).

Mutations in USP8 hot spot occur in the region involved in binding 14-3-3 proteins and thus impair interactions between USP8 and 14-3-3 proteins (33). As a consequence, the activity of USP8 is enhanced leading to deubiquitination of its substrates and preventing their proteasomal degradation. This mechanism was clearly shown in vitro providing the explanation of the sustained EGFR signalling in corticotroph tumours with USP8 mutations $(3,4)$. However, there is also evidence that the level of other proteins associated with tumour development such as MET1 (34) and Cdt2 (35) may be sustained with this mechanism. Regulatory role of USP8 in preventing degradation of VEGFR2 (36), smoothened (SMO) (37), frizzled (FZD) (38) and Nrdp1 (39) was also reported. The large number of DEGs and the enriched pathways in comparison of USP8-mutated and wild-type tumours suggest that the mutation exerts a pleiotropic biological effect not limited to EGFR pathway that it is manifested by deregulation of many proteins. When comparing USP8mut and USP8wt patients we did not observe difference in USP8 and EGFR expression levels, as also reported previously $(4,24)$. We also did not find EGFR-related pathways as significantly enriched for DEGs in GSE analysis.

According to our study and previous research (4, 24) USP8mut tumours show higher POMC expression, which is concordant with the reported increase in ACTH expression caused by enhanced activity of $P O M C$ promoter in USP8mut tumours (3).

We have paid special attention to comparison of gene expression profiles of SCAs and functioning corticotroph tumours causing CD. The biological differences between functioning and non-functioning corticotroph tumours have been investigated in very few studies. Intriguingly, the cause of the silent nature of SCAs remains elusive. Unexpectedly, our results show that the functionally active and silent corticotrophinomas differ only slightly in terms of genes expression with only 66 DEGs (with FC $>2.5$ ) found in this comparison. The identified genes are involved in cell signalling (GLI2, DLC1, TBX2, RASSF6), cell adhesion (GJA1, CDH6) or ion channels functioning (KCNN4, KCNJ5). We did not find significant differences in 
the expression of genes related to ACTH release including prohormone convertase $1 / 3$ gene $(9,10,12)$, SSTR1, SSTR2, CRHR1, AVPR1B (V1BR), HSD11B2 $(7,12)$ or genes related to differentiation of pituitary cells NEUROD1 and TBX19 (TPIT) that have been reported previously as differentially expressed in SCAs and functioning corticotrophinomas $(7,12)$.

The physiological activity of pituitary cells is regulated by changes in membrane potential. Secretory activity of corticotroph cells is provoked by membrane depolarization associated with the opening of $\mathrm{Ca}^{2+}$ channels and the influx of these ions into the cell (40). Therefore, the genes involved in electrical excitability of cells are of a special interest when comparing SCA and functioning tumours. Indeed, genes encoding ion channels (KCNJ5, KCNN4, GABRD), as well as other genes involved in regulation of membrane potential (GABBR2, GNAL, GJA1 - connexin 43) were found differentially expressed.

KCNJ5 was identified as a DEG with the highest fold change of expression between SCAs and CD adenomas. It encodes KIR3.4 (GIRK4) protein, which is a G-proteinactivated inward rectifier potassium channel. KIR3 family channels are involved in stabilization of resting membrane potential (RMP) and prevent membrane hyperpolarization. Blocking $\mathrm{K}+$ channels results in membrane depolarization and increase in $\mathrm{Ca} 2+$ concentration that triggers $\mathrm{ACTH}$ secretion in corticotroph cells (41). KIR channels were shown to be involved in maintaining RMP in corticotroph cells and their inhibition contributes to firing activation potential upon CRH stimulation (42).

Four KIR3 channels have been identified in humans. KIR3-encoding genes are mostly expressed in brain and pituitary, with exception of KIR3.4 (KCNJ5) that is mostly expressed in adrenal gland and pituitary (based on GtEX database) (43). In pituitary, KCNJ5 has the highest expression of all KIR3-encoding genes (based on GtEX database). In adrenal gland KIR3.4 seems to play a pivotal role in aldosterone secretion. Somatic KCNJ5 mutations are found in aldosterone-producing adenomas, while germline mutations of this gene cause familial hyperaldosteronism type 3. KCNJ5 mutations result in loss of selectivity for $\mathrm{K}+$, causing $\mathrm{Na}+$ influx, calcium mobilization, increased depolarization, and hyperaldosteronism (44). KIR3 channels are downstream effectors of G-protein-coupled receptors (45). As observed in corticotroph AtT-20 cells, stimulation of somatostatin receptor $(46,47)$ and dopamine receptors (48) results in activation of KIR channels. Since KIR channels are involved in a negative regulation of ACTH secretion, high expression of KCNJ5 in SCAs can be considered as functionally related to inactive nature of these tumours.

When GSE method was applied for the analysis of genes differentially expressed between SCAs and functioning corticotrophinomas, the enrichment in the processes of activation of GABA receptor $B$ (GABARB) was the only pathway found in Reactome database. This Reactome pathway includes three DEGs: KCNJ5 (described above) as well as GABBR2 and GNAL, both with higher expression in functioning tumours. Additionally, CD-causing tumours showed also higher expression of another gene related to GABA signalling - GABRD. In anterior pituitary GABA acts in a paracrine and autocrine manner, and it stimulates some functional subtypes of the cells including corticotrophs, while it has inhibitory effect on other ones $(49,50,51)$. Accordingly to this general role of GABA, we observed the higher expression of genes encoding subunits of GABA receptors $\mathrm{B}(G A B B R 2)$ and $\mathrm{A}(G A B R D)$ in functioning corticotroph tumours than in SCAs. The possible functional consequences of these results are unclear. GABARB subunits are expressed in corticotroph cells $(50,51)$, but the available data indicate that this receptor does not play a role in ACTH release $(49,51$, 52). In corticotroph cells, hormone secretion is mediated by stimulating GABA receptor A (GABARA) rather than GABARB, as the cells release ACTH upon stimulation with muscimol, and not with baclofen $(46,48)$. On the other hand, GABARA receptor is a large pentameric ligandgated ion channel consisting of five subunits of different types $(\alpha 1-6, \beta 1-3, \gamma 1-3, \delta, \varepsilon, \pi$ and $\theta)$ and only the gene encoding subunit $\delta(G A B R D)$ is expressed at higher level in functioning adenomas than in SCAs. It is not clear how overexpression of one subunit only would affect receptor function. It was shown that stimulation of $\delta$ subunit with specific agonist enhances secretory activity of pituitary gonadotrophs (53), but no data regarding the role of this subunit in corticotroph cells are available.

According to GSE analysis with Reactome, KCNJ5 and GNAL play a role in GABARB signalling. However, considering the reports of no influence of GABARB stimulation on ACTH release, it is fairly probable that KCNJ5 and GNAL (encoding G-protein subunit alpha) act as effectors for other G-protein-coupled receptors. The role of GABA signalling has been most extensively studied in adult neurons, where KIR channels are one of the effectors for $G A B A B$ receptor (54). In corticotroph tumours we observed the inverse correlation of expression of GABBR2 and $K C N J 5$ that suggest the opposite role of these genes.

In summary, our results show that USP8 hot spot mutations occur in corticotroph adenomas causing CD as 
well as in SCAs albeit with low frequency. USP8 mutations determine the molecular profile of the tumour. They have a pleiotropic effect affecting the expression levels of numerous genes involved in different molecular processes. Expression levels of relatively low number of genes were found to distinguish SCAs and CD-causing tumours. Our findings suggest that high KCNJ5 expression may be involved in lowering electrical excitability of the cells in SCA, while high expression levels of genes involved in GABA signalling may favour ACTH hypersecretion in tumours causing CD.

\section{Supplementary data}

This is linked to the online version of the paper at https://doi.org/10.1530/ EJE-19-0194.

\section{Declaration of interest}

The authors declare that there is no conflict of interest that could be perceived as prejudicing the impartiality of this study.

\section{Funding}

Supported by Maria Sklodowska-Curie Institute - Oncology Center internal grant SN/GW09/2017.

\section{Acknowledgements}

The authors thank M Kowalewska and A Kiepurski for proofreading the manuscript.

\section{References}

1 Ben-Shlomo A \& Cooper O. Silent corticotroph adenomas. Pituitary 201821 1-9. (doi:10.1007/s11102-018-0864-8)

2 Albani A, Theodoropoulou M \& Reincke M. Genetics of Cushing's disease. Clinical Endocrinology 201888 3-12. (https://doi. org/10.1111/cen.13457)

3 Reincke M, Sbiera S, Hayakawa A, Theodoropoulou M, Osswald A, Beuschlein F, Meitinger T, Mizuno-Yamasaki E, Kawaguchi K, Saeki Y et al. Mutations in the deubiquitinase gene USP8 cause Cushing's disease. Nature Genetics 201547 31-38. (https://doi.org/10.1038/ ng.3166)

4 Ma ZY, Song ZJ, Chen JH, Wang YF, Li SQ, Zhou LF, Mao Y, Li YM, Hu RG, Zhang ZY et al. Recurrent gain-of-function USP8 mutations in Cushing's disease. Cell Research 201525 306-317. (https://doi. org/10.1038/cr.2015.20)

5 Perez-Rivas LG, Theodoropoulou M, Ferraù F, Nusser C, Kawaguchi K, Stratakis CA, Faucz FR, Wildemberg LE, Assié G, Beschorner R et al. The gene of the ubiquitin-specific protease 8 is frequently mutated in adenomas causing Cushing's disease. Journal of Clinical Endocrinology and Metabolism 2015100 E997-E1004. (https://doi.org/10.1210/ jc.2015-1453)

6 Gabalec F, Beranek M, Netuka D, Masopust V, Nahlovsky J, Cesak T, Marek J \& Cap J. Dopamine 2 receptor expression in various pathological types of clinically non-functioning pituitary adenomas Pituitary 201215 222-226. (https://doi.org/10.1007/s11102-0110316-1)
7 Tateno T, Kato M, Tani Y, Oyama K, Yamada S \& Hirata Y. Differential expression of somatostatin and dopamine receptor subtype genes in adrenocorticotropin (ACTH)-secreting pituitary tumors and silent corticotroph adenomas. Endocrine Journal 200956 579-584. (https:// doi.org/10.1507/endocrj.k08e-186)

8 Righi A, Faustini-Fustini M, Morandi L, Monti V, Asioli S, Mazzatenta D, Bacci A \& Foschini MP. The changing faces of corticotroph cell adenomas: the role of prohormone convertase $1 / 3$. Endocrine 201756 286-297. (https://doi.org/10.1007/s12020-0161028-0)

9 Ohta S, Nishizawa S, Oki Y, Yokoyama T \& Namba H. Significance of absent prohormone convertase $1 / 3$ in inducing clinically silent corticotroph pituitary adenoma of subtype I immunohistochemical study. Pituitary 20025 221-223. (https://doi. org/10.1023/A:1025321731790)

10 Tateno T, Izumiyama H, Doi M, Akashi T, Ohno K \& Hirata Y. Defective expression of prohormone convertase $1 / 3$ in silent corticotroph adenoma. Endocrine Journal 200754 777-782. (https:// doi.org/10.1507/endocrj.k07-059)

11 Nagaya T, Seo H, Kuwayama A, Sakurai T, Tsukamoto N, Nakane T, Sugita K \& Matsui N. Pro-opiomelanocortin gene expression in silent corticotroph-cell adenoma and Cushing's disease. Journal of Neurologicalsurgery 199072 262-267. (https://doi.org/10.3171/ jns.1990.72.2.0262)

12 Tateno T, Izumiyama H, Doi M, Yoshimoto T, Shichiri M, Inoshita N, Oyama K, Yamada S \& Hirata Y. Differential gene expression in ACTH-secreting and non-functioning pituitary tumors. European Journal of Endocrinology 2007157 717-724. (https://doi.org/10.1530/ EJE-07-0428)

13 Raverot G, Wierinckx A, Jouanneau E, Auger C, Borson-Chazot F, Lachuer J, Pugeat M \& Trouillas J. Clinical, hormonal and molecular characterization of pituitary ACTH adenomas without (silent corticotroph adenomas) and with Cushing's disease. European Journal of Endocrinology 2010163 35-43. (https://doi.org/10.1530/EJE-100076)

14 Di Meo A, Rotondo F, Kovacs K, Cusimano MD, Syro LV, Di Ieva A, Diamandis EP \& Yousef GM. Human kallikrein 10 expression in surgically removed human pituitary corticotroph adenomas: an immunohistochemical study. Applied Immunohistochemistry and Molecular Morphology 201523 433-437. (https://doi.org/10.1097/ PAI.0000000000000108)

15 Tani Y, Inoshita N, Sugiyama T, Kato M, Yamada S, Shichiri M \& Hirata Y. Upregulation of CDKN2A and suppression of cyclin D1 gene expressions in ACTH-secreting pituitary adenomas. European Journal of Endocrinology 2010163 523-529. (https://doi.org/10.1530/EJE-10-0245)

16 Thodou E, Argyrakos T \& Kontogeorgos G. Galectin-3 as a marker distinguishing functioning from silent corticotroph adenomas. Hormones 20076 227-232.

17 Salehi F, Scheithauer BW, Kovacs K, Horvath E, Syro LV, Sharma S, Manoranjan B \& Cusimano M. O-6-methylguanine-DNA methyltransferase (MGMT) immunohistochemical expression in pituitary corticotroph adenomas. Neurosurgery 201270 491-496; discussion 496. (https://doi.org/10.1227/NEU.0b013e318230ac63)

18 Lawrence M, Huber W, Pagès H, Aboyoun P, Carlson M, Gentleman R, Morgan MT \& Carey VJ. Software for computing and annotating genomic ranges. PLoS Computational Biology 20139 e1003118. (https://doi.org/10.1371/journal.pcbi.1003118)

19 Love MI, Huber W \& Anders S. Moderated estimation of fold change and dispersion for RNA-seq data with DESeq2. Genome Biology 2014 15 550. (https://doi.org/10.1186/s13059-014-0550-8)

20 McCall MN, McMurray HR, Land H \& Almudevar A. On non-detects in qPCR data. Bioinformatics 201430 2310-2316. (https://doi. org/10.1093/bioinformatics/btu239)

21 McCarty Jr KS, Szabo E, Flowers JL, Cox EB, Leight GS, Miller L, Konrath J, Soper JT, Budwit DA \& Creasman WT. 
Use of a monoclonal anti-estrogen receptor antibody in the immunohistochemical evaluation of human tumors. Cancer Research 198646 4244s-4248s.

22 Kuleshov MV, Jones MR, Rouillard AD, Fernandez NF, Duan Q Wang Z, Koplev S, Jenkins SL, Jagodnik KM, Lachmann A et al. Enrichr: a comprehensive gene set enrichment analysis web server 2016 update. Nucleic Acids Research 201644 W90-W97. (https://doi. org/10.1093/nar/gkw377)

23 Faucz FR, Tirosh A, Tatsi C, Berthon A, Hernández-Ramírez LC, Settas N, Angelousi A, Correa R, Papadakis GZ, Chittiboina P et al. Somatic USP8 gene mutations are a common cause of pediatric Cushing disease. Journal of Clinical Endocrinology and Metabolism 2017 102 2836-2843. (https://doi.org/10.1210/jc.2017-00161)

24 Hayashi K, Inoshita N, Kawaguchi K, Ardisasmita AI, Suzuki H, Fukuhara N, Okada M, Nishioka H, Takeuchi Y, Komada M et al. The USP8 mutational status may predict drug susceptibility in corticotroph adenomas of Cushing's disease. European Journal of Endocrinology 2016174 213-226. (https://doi.org/10.1530/EJE-150689)

25 Ballmann C, Thiel A, Korah HE, Reis AC, Saeger W, Stepanow S, Köhrer K, Reifenberger G, Knobbe-Thomsen CB, Knappe UJ et al. USP8 mutations in pituitary Cushing adenomas-targeted analysis by next-generation sequencing. Journal of the Endocrine Society 20182 266-278. (https://doi.org/10.1210/js.2017-00364)

26 Losa M, Mortini P, Pagnano A, Detomas M, Cassarino MF \& Pecori Giraldi F. Clinical characteristics and surgical outcome in USP8mutated human adrenocorticotropic hormone-secreting pituitary adenomas. Endocrine 201963 240-246. (https://doi.org/10.1007/ s12020-018-1776-0)

27 Weigand I, Knobloch L, Flitsch J, Saeger W, Monoranu CM, Höfner K, Herterich S, Rotermund R, Ronchi CL, Buchfelder M et al. Impact of USP8 gene mutations on protein deregulation in Cushing disease. Journal of Clinical Endocrinology and Metabolism 2019104 2535-2546. (https://doi.org/10.1210/jc.2018-02564)

28 Zoli M, Faustini-Fustini M, Mazzatenta D, Marucci G, De Carlo E, Bacci A, Pasquini E, Lanzino G \& Frank G. ACTH adenomas transforming their clinical expression: report of 5 cases. Neurosurgical Focus 201538 E15. (https://doi.org/10.3171/2014.11. FOCUS14679)

29 Singh R, Bassett E, Chakravarti A \& Parthun MR Replicationdependent histone isoforms: a new source of complexity in chromatin structure and function. Nucleic Acids Research 201846 8665-8678. (https://doi.org/10.1093/nar/gky768)

30 Araki T \& Liu NA. Cell cycle regulators and lineage-specific therapeutic targets for Cushing disease. Frontiers in Endocrinology 20189 444. (https://doi.org/10.3389/fendo.2018.00444)

31 Sur S \& Agrawal DK. Phosphatases and kinases regulating CDC25 activity in the cell cycle: clinical implications of CDC25 overexpression and potential treatment strategies. Molecular and Cellular Biochemistry 2016416 33-46. (https://doi.org/10.1007/ s11010-016-2693-2)

32 Wang W, Shen T, Dong B, Creighton CJ, Meng Y, Zhou W, Shi Q, Zhou H, Zhang Y, Moore DD et al. MAPK4 overexpression promotes tumor progression via noncanonical activation of AKT/mTOR signaling. Journal of Clinical Investigation 2019129 1015-1029. (https://doi.org/10.1172/JCI97712)

33 Centorrino F, Ballone A, Wolter M \& Ottmann C. Biophysical and structural insight into the USP8/14-3-3 interaction. FEBS Letters 2018 592 1211-1220. (https://doi.org/10.1002/1873-3468.13017)

34 Kim Y, Shiba-Ishii A, Nakagawa T, Iemura SI, Natsume T, Nakano N, Matsuoka R, Sakashita S, Lee SJ, Kawaguchi A et al. Stratifin regulates stabilization of receptor tyrosine kinases via interaction with ubiquitin-specific protease 8 in lung adenocarcinoma. Oncogene 2018 37 5387-5402. (https://doi.org/10.1038/s41388-018-0342-9)

35 Dar A, Wu D, Lee N, Shibata E \& Dutta A. 14-3-3 proteins play a role in the cell cycle by shielding cdt 2 from ubiquitin-mediated degradation. Molecular and Cellular Biology 201434 4049-4061. (https://doi.org/10.1128/MCB.00838-14)

36 Smith GA, Fearnley GW, Abdul-Zani I, Wheatcroft SB, Tomlinson DC, Harrison MA \& Ponnambalam S. VEGFR2 trafficking, signaling and proteolysis is regulated by the ubiquitin isopeptidase USP8. Traffic 201617 53-65. (https://doi.org/10.1111/tra.12341)

37 Xia R, Jia H, Fan J, Liu Y \& Jia J. USP8 promotes smoothened signaling by preventing its ubiquitination and changing its subcellular localization. PLoS Biology 201210 e1001238. (https://doi. org/10.1371/journal.pbio.1001238)

38 Mukai A, Yamamoto-Hino M, Awano W, Watanabe W, Komada M \& Goto S. Balanced ubiquitylation and deubiquitylation of Frizzled regulate cellular responsiveness to Wg/Wnt. EMBO Journal 201029 2114-2125. (https://doi.org/10.1038/emboj.2010.100)

39 Wu X, Yen L, Irwin L, Sweeney C \& Carraway III KL. Stabilization of the E3 ubiquitin ligase Nrdp1 by the deubiquitinating enzyme USP8. Molecular and Cellular Biology 200424 7748-7757. (https://doi. org/10.1128/MCB.24.17.7748-7757.2004)

40 Zemkova H, Tomić M, Kucka M, Aguilera G \& Stojilkovic SS. Spontaneous and CRH-induced excitability and calcium signaling in mice corticotrophs involves sodium, calcium, and cationconducting channels. Endocrinology 2016157 1576-1589. (https:// doi.org/10.1210/en.2015-1899)

41 Wang X \& Greer MA. Blocking K+ channels with TEA induces plasmalemma depolarization, increased [Ca2+]i, and ACTH secretion in AtT-20 cells. Molecular and Cellular Endocrinology 1995109 11-18. (https://doi.org/10.1016/0303-7207(95)03478-p)

42 Kuryshev YA, Haak L, Childs GV \& Ritchie AK. Corticotropin releasing hormone inhibits an inwardly rectifying potassium current in rat corticotropes. Journal of Physiology $1997 \mathbf{5 0 2} 265-279$. (https:// doi.org/10.1111/j.1469-7793.1997.265bk.x)

43 Lonsdale J, Thomas J, Salvatore M, Phillips R, Lo E, Shad S, Hasz R, Walters G, Garcia F, Young N et al. The Genotype-Tissue Expression (GTEx) project. Nature Genetics 201345 580-585. (https://doi. org/10.1038/ng.2653)

44 Gomez-Sanchez CE \& Oki K. Minireview: potassium channels and aldosterone dysregulation: is primary aldosteronism a potassium channelopathy? Endocrinology 2014155 47-55. (https://doi. org/10.1210/en.2013-1733)

45 Stojilkovic SS, Tabak J \& Bertram R. Ion channels and signaling in the pituitary gland. Endocrine Reviews 201031 845-915. (https:// doi:10.1210. Engineeringresearch 2010-0005).

46 Tallent M, Dichter MA \& Reisine T. Evidence that a novel somatostatin receptor couples to an inward rectifier potassium current in AtT-20 cells. Neuroscience 199673 855-864. (https://doi. org/10.1016/0306-4522(96)00079-6)

47 Takano K, Yasufuku-Takano J, Kozasa T, Nakajima S \& Nakajima Y. Different $G$ proteins mediate somatostatin-induced inward rectifier K+currents in murine brain and endocrine cells. Journal of Physiology $1997 \mathbf{5 0 2} 559-567$. (https://doi.org/10.1111/j.14697793.1997.559bj.x)

48 Kuzhikandathil EV, Yu W \& Oxford GS. Human dopamine D3 and D2L receptors couple to inward rectifier potassium channels in mammalian cell lines. Molecular and Cellular Neurosciences 199812 390-402. (https://doi.org/10.1006/mcne.1998.0722)

49 Zemkova HW, Bjelobaba I, Tomic M, Zemkova H \& Stojilkovic SS. Molecular, pharmacological and functional properties of GABA(A) receptors in anterior pituitary cells. Journal of Physiology $2008 \mathbf{5 8 6}$ 3097-3111. (https://doi.org/10.1113/jphysiol.2008.153148)

50 Mayerhofer A, Höhne-Zell B, Gamel-Didelon K, Jung H, Redecker P, Grube D, Urbanski HF, Gasnier B, Fritschy JM \& Gratzl M. Gammaaminobutyric acid (GABA): a para- and/or autocrine hormone in the pituitary. FASEB Journal 200115 1089-1091. (https://doi. org/10.1096/fj.00-0546fje)

51 Anderson RA \& Mitchell R. Effects of gamma-aminobutyric acid receptor agonists on the secretion of growth hormone, 
luteinizing hormone, adrenocorticotrophic hormone and thyroidstimulating hormone from the rat pituitary gland in vitro. Journal of Endocrinology 1986108 1-8. (https://doi.org/10.1677/joe.0.1080001)

52 Pitman KA, Borgland SL, MacLeod B \& Puil E. Isovaline does not activate GABA(B) receptor-coupled potassium currents in GABA(B) expressing AtT-20 cells and cultured rat hippocampal neurons. PLOS ONE 201510 e0118497. (https://doi.org/10.1371/journal. pone.0118497)
53 Mijiddorj T, Kanasaki H, Sukhbaatar U, Oride A \& Kyo S. DS1, a delta subunit-containing $\mathrm{GABA}(\mathrm{A})$ receptor agonist, increases gonadotropin subunit gene expression in mouse pituitary gonadotrophs. Biology of Reproduction 201592 45. (https://doi. org/10.1095/biolreprod.114.123893)

54 Padgett CL \& Slesinger PA. GABAB receptor coupling to G-proteins and ion channels. Advances in Pharmacology 201058 123-147. (https://doi.org/10.1016/S1054-3589(10)58006-2)

Received 14 March 2019

Revised version received 5 September 2019

Accepted 2 October 2019 